\title{
Stable Robust Adaptive Control of Robotic Manipulators with Switched Constraints
}

\author{
Ibrahim F. Jasim \\ Faculty of Science, Technology, and Communication \\ University of Luxembourg \\ L-1359 Luxembourg \\ E-mail: ibrahim.jasim@uni.lu
}

\author{
Peter W. Plapper \\ Faculty of Science, Technology, and Communication \\ University of Luxembourg \\ L-1359 Luxembourg \\ Email: peter.plapper@uni.lu
}

\begin{abstract}
In this paper, the problem of controlling switched constrained robotic manipulators is addressed. Switched constrained robots are those robots interacting with multiple switched constraints. We start our control algorithm with suggesting a sliding mode controller that is proved to provide stable system performance. However, the bounds of the functions, on each link, caused from the constraints are assumed to be known. Then an adaptive sliding mode control strategy is suggested that relaxes the need for knowing the bounds of the constraints functions with guaranteeing global stable performance of the given switched constrained robotic system. Finally, we complement the control strategy above through deriving an improved robust adaptive control scheme that is proved to give a stable performance with reduced chattering. All of the three stages of the suggested control strategy are derived through finding a common Lyapunov function that can stabilize all of the subsystems for the overall switched system. Simulation is carried out for a two link robotic manipulator interacting with two switched constraints. From the simulation results we can see the excellent tracking performance and the high efficiency of the suggested control strategy in controlling switched constrained robotic systems.
\end{abstract}

Index Terms- Constrained motion robots; robust adaptive control; sliding mode control; switched systems.

\section{INTRODUCTION}

Due to their diversified applications, robots were attracted by practitioners from both academia and industry of robotics and control. Different approaches were suggested in having a stable control performance. In [1,2] , PD and PID controllers were used in controlling robots. Adaptive control strategies were successfully employed in having an enhanced control performance [3,4]. Hybrid force/position control strategies were successfully used in handling the control problem of constrained motion robotic manipulators [5-8]. In [9], adaptive fuzzy control scheme was suggested to handle the control problem of constrained robots with holonomic and nonholonomic constraints. The passivity property of the robots was successfully employed in improving the control strategy of robotic systems $[10,11]$. For repetitive robotic tasks, a switched control strategy was suggested in the sense of iteration, i.e. the switching between different sub-controllers is done according to the iteration index [12]. Other strategies were suggested to improve the robots control strategy more, like handling the time varying parameters in the robots [13], deadzone and backlash existing at the links actuation [14-16]. However, a common feature to the approaches suggested so far is the lack of handling possible switching in the robot dynamics that may limit the performance significantly. In [17], it was shown that for switched control systems, switching between different stable subsystems can cause unstable performance. In many robots applications, like for instance robotic assembly, the dynamics is fixed but the constraints are changing that would add a switched term to the robot dynamics caused from the switched constraints.

In this paper, we address the problem of controlling robots with switched constraints. Developing the control strategy will be composed of three stages. In the first stage, a sliding mode control scheme is suggested that requires the knowledge of the bounds of the functions resulted from the constraints for each link. In the second stage, we relax the requirement of knowing the bounds of the constraints functions through developing an adaptive sliding mode control scheme in which those bounds are compensated on-line. Finally, we suggest a robust adaptive control strategy so that we can avoid possible chattering in the strategy got from the second stage. All stages of the design will be proved to have stable performance through finding a common Lyapunov function that can act as a stability measure for all of the subsystems of the the overall switched robotic system.

The rest of the paper is organized as follows. In section 2, we describe the dynamic equation of the robotic manipulators with switched constraints. The main results along with the control strategies are explained in section 3. In section 4, simulation is performed and section 5 gives the concluding remarks.

\section{PROBLEM FORMULATION}

The dynamic equations of a constrained robotic system can be described by $[18,19]$ :

$$
M(q) \ddot{q}+C(q, \dot{q}) \dot{q}+G(q)=\tau+J^{T}(q) D^{T}(\alpha) \lambda
$$

Where $q \in R^{n}$ is the links position vector, $M(q) \in R^{n \times n}$ is the inertia matrix, $C(q, \dot{q}) \dot{q} \in R^{n}$ is the centripetal and Coriolis vector, $G(q) \in R^{n \times n}$ is the gravity vector, $\tau \in R^{n}$ is the torque vector actuating the links, $J(q) \in R^{n \times 6}$ is the Jacobian of the manipulator, $\lambda \in R^{m}$ is the vector of Lagrange multipliers, and $D(\alpha)$ is the gradient of the task space constraints, with $\alpha$ is the pose of the end effector which is related to the joint space as:

$$
\alpha=H(q)
$$


$H: R^{n} \rightarrow R^{6}$ is the homogenous transformation matrix that maps the generalized variables of the robot to the task space. The above homogenous transformation matrix is for the three dimensional space, and the mapping dimensions for the case of a two dimensional task space would be $H: R^{n} \rightarrow R^{4}$ with $\alpha \in R^{4}$. The constraints of the robot can be described by:

$$
\Phi(\alpha)=0
$$

$\Phi(\alpha)=\left[\phi_{1}(\alpha), \phi_{2}(\alpha), \ldots, \phi_{m}(\alpha)\right], \Phi(\alpha): R^{n} \rightarrow R^{m}$ is the kinematic constraints due to the environment. The expression of $D(\alpha)$ can be written as:

$$
D(\alpha)=\frac{\partial \Phi(\alpha)}{\partial \alpha}
$$

For the case of multiple switching constraints, (4) can be written as:

$$
D_{\sigma}(\alpha)=\frac{\partial \Phi_{\sigma}(\alpha)}{\partial \alpha}
$$

$\sigma$ is the index of the constraints $(\sigma=1,2, \ldots, P), \mathrm{P}$ is the total number of constraints. Substituting (5) into (1), then the dynamics of a robotic system with multiple switching constraints can be written as:

$$
M(q) \ddot{q}+C(q, \dot{q}) \dot{q}+G(q)=\tau+J^{T}(q) D_{\sigma}^{T}(\alpha) \lambda
$$

Equation (6) is a nonlinear switched system, and the objective of this paper is to propose a control strategy that can guarantee stable performance of the robot under such a switching behavior. In the next section, we will present the main results obtained in this paper.

\section{MAIN RESULTS}

Equation (6) can be rewritten in the following form:

$$
M(q) \ddot{q}+C(q, \dot{q}) \dot{q}+G(q)=\tau+f_{\sigma}(\alpha)
$$

where:

$$
f_{\sigma}(\alpha)=J^{T}(q) D_{\sigma}^{T}(\alpha) \lambda
$$

For all robotic systems, the properties below can be noticed [10]:

P1. For all robotic manipulators, $M(q)$ is a positive definite and symmetric matrix.

P2. For all robotic manipulators, the matrix $\dot{M}(q)-2 C(q, \dot{q})$ is a skew symmetric matrix, that is for all $x \neq 0$, we have $x^{T}(\dot{M}(q)-2 C(q, \dot{q})) x=0$.

Define the joints error vector to be:

$$
\tilde{q}=q-q_{d}
$$

Consider the joints filtered error vector to be described as:

$$
s=\dot{\tilde{q}}+\gamma \tilde{q}
$$

with $\gamma>0$. (10) can be rewritten as:

$$
s=\dot{q}-\dot{q_{r}}
$$

where:

$$
\dot{q}_{r}=\dot{q}_{d}-\gamma \tilde{q}
$$

Note 1. It has been shown that the filtered error described by (10) has the following properties: (i) the equation $s(t)=0$ defines the time-varying hyperplane in $R^{n}$, on which the tracking error vector $\tilde{q}$ decays exponentially to zero.(ii) if $\tilde{q}(0)=0$ and $|s(t)| \leq \varepsilon$ with constant $\varepsilon$, then $\tilde{q}(t) \in \Omega_{\varepsilon}=$ $\left\{\frac{\tilde{q}(t)}{\tilde{q}_{i}} \leq 2^{i-1} \gamma^{i-2} \varepsilon, i=1,2\right\}$ for $\forall t \geq 0$ and (iii) if $\tilde{q}(0) \neq 0$ and $|s(t)| \leq \varepsilon$ then $\tilde{q}(t)$ will converge to $\Omega_{\varepsilon}$ within a time constant of $\frac{(n-1)}{\gamma}[20,21]$.

Taking the time derivative of (11), we obtain:

$$
\dot{s}=\ddot{q}-\ddot{q}_{r}
$$

In order to derive a control strategy that can guarantee the stability of (7) for all phases of such a switched nonlinear system, we need to find a common Lyapunov function for all phases which can assure that the suggested control law is stabilizing all the phases of the switched system [17]. To derive such a control law, suppose that the upper bound of the function $f_{\sigma}(q)$ is known, that is:

$$
\left|f_{\sigma}(q)\right| \leq B_{\sigma}
$$

where $B_{\sigma}$ is assumed to be known. Furthermore, the desired joint angles $q_{d}$ with their $1^{\text {st }}$ and $2^{\text {nd }}$ derivatives are assumed to be piecewise continuous. As a summary, the following two assumptions are considered:

A1. The signals $q_{d}, \dot{q}_{d}$, and $\ddot{q}_{d}$ are assumed to be bounded and piecewise continuous.

A2. The bounds of the constraint functions $B_{\sigma}(q)$ are assumed to be known.

Then the theorem below can be deduced for the given switched constrained robotic system.

Theorem 1: For the robotic system described by (7) and satisfying assumptions $\mathrm{A} 1$ and $\mathrm{A} 2$, the control law:

$$
\tau=C(q, \dot{q}) \dot{q}_{r}+G(q)+M(q) \ddot{q}_{r}-B \operatorname{sgn}(s)-k_{d} s
$$

can guarantee global stable performance with all closed loop signals are guaranteed to be bounded. Where $k_{d} \geq 0$ and:

$$
B=\sup _{\sigma}\left(B_{\sigma}\right)
$$

Proof: Consider the Lyapunov candidate:

$$
V=\frac{1}{2} s^{T} M(q) s
$$

Taking the time derivative of (17), we obtain:

$$
\dot{V}=s^{T} M(q) \dot{s}+\frac{1}{2} s^{T} \dot{M}(q) s
$$

Substituting (13) into (18), we get:

$$
\begin{gathered}
\dot{V}=s^{T} M(q)\left(\ddot{q}-\ddot{q}_{r}\right)+\frac{1}{2} s^{T} \dot{M}(q) s \\
\dot{V}=s^{T}\left(M(q) \ddot{q}-M(q) \ddot{q}_{r}\right)+\frac{1}{2} s^{T} \dot{M}(q) s
\end{gathered}
$$

From (7), we have:

$$
M(q) \ddot{q}=\tau+f_{\sigma}(q)-C(q, \dot{q}) \dot{q}-G(q)
$$

Substituting (21) into (20) results:

$$
\begin{array}{r}
\dot{V}=s^{T}\left(\tau+f_{\sigma}(q)-C(q, \dot{q}) \dot{q}-G(q)-M(q) \ddot{q}_{r}\right)+ \\
\frac{1}{2} s^{T} \dot{M}(q) s
\end{array}
$$


From (11), we have $\dot{q}=s+\dot{q}_{r}$, therefore (22) can be written as:

$$
\begin{array}{r}
\dot{V}=s^{T}\left(\tau+f_{\sigma}(q)-C(q, \dot{q})\left(s+\dot{q}_{r}\right)-G(q)-M(q) \ddot{q}_{r}\right) \\
+\frac{1}{2} s^{T} \dot{M}(q) s
\end{array}
$$

Using property $\mathrm{P} 2$ and after several mathematical manipulations, (23) can be rewritten as:

$$
\dot{V}=s^{T}\left(\tau+f_{\sigma}(q)-C(q, \dot{q}) \dot{q}_{r}-G(q)-M(q) \ddot{q}_{r}\right)
$$

Using the control action (15) into (24), we obtain:

$$
\begin{gathered}
\dot{V}=s^{T}\left(-B \operatorname{sgn}(s)-k_{d} s+f_{\sigma}(q)\right) \\
\dot{V}=-B s^{T} \operatorname{sgn}(s)-k_{d} s^{T} s+s^{T} f_{\sigma}(q) \\
\dot{V}=-B\left|s^{T}\right|-k_{d} s^{T} s+s^{T} f_{\sigma}(q)
\end{gathered}
$$

From (14) and (16), we can have:

$$
-B\left|s^{T}\right|+s^{T} f_{\sigma}(q) \leq 0
$$

Therefore, from (27) and (28) we obtain:

$$
\dot{V} \leq-k_{d} s^{T} s
$$

Integrating both sides of (29) results:

$$
\int_{0}^{t} k_{d} s^{T} s \leq V(0)-V(t)<\infty
$$

Therefore, $s \in \mathcal{L}_{2} \cap \mathcal{L}_{\infty}$ and from (10) we can conclude that $\tilde{q}$ is bounded. So, from assumption A1, we can conclude that $q$ is also bounded. From (11), we can say that $\dot{s}$ is bounded, that is $\dot{s} \in \mathcal{L}_{\infty}$. Since we have $s \in \mathcal{L}_{2} \cap \mathcal{L}_{\infty}$ and $\dot{s} \in \mathcal{L}_{\infty}$, then according to Barbalats lemma $s \rightarrow 0$ as $t \rightarrow \infty$ which implies that $\tilde{q} \rightarrow 0$ as $t \rightarrow \infty$. So, the control action $\tau$ described by (15) is also bounded.

So, (17) is really a common Lyapunov function for all subsystems of the overall constrained switched robotic system described by (7). Furthermore, we can see that the term $B \operatorname{sgn}(s)$ in (15) compensates for the existence of the term $J^{T}(q) D^{T}(\alpha) \lambda$ resulted from the constraints. Despite the asymptotic convergence of the control law given in (15), it requires the knowledge of the bounds of the functions $f_{\sigma}(q)$ which is difficult to be found. In the control strategy given in theorem 2 below, we will relax assumption A2 and the bounds of $f_{\sigma}(q)$ will be on-line adapted and as detailed in the theorem below.

Theorem 2: For the constrained robotic system given in (7) and satisfying assumption A1 the control law:

$$
\tau=C(q, \dot{q}) \dot{q}_{r}+G(q)+M(q) \ddot{q}_{r}-\hat{B} \operatorname{sgn}(s)-k_{d} s
$$

with the bounds parameters update law:

$$
\dot{\hat{B}}= \begin{cases}\eta|s| & \text { if }\left(|\hat{B}|<M_{B}\right) \text { or }\left(\hat{B}=M_{B} \text { and } \eta|s| \leq 0\right) \\ P(\eta|s|) & \text { if }\left(\hat{B}=M_{B} \text { and } \eta|s|>0\right)\end{cases}
$$

can guarantee global stable performance with all closed loop signals are guaranteed to be bounded. Where $\eta, k_{d} \geq 0, M_{B}$ is a design parameter vector, and $P($.$) is the projection function,$ that is:

$$
P(\eta|s|)=\eta|s|-\eta|s|\left(\frac{\hat{B}^{T} \hat{B}}{|\hat{B}|^{2}}\right)
$$

Proof: Consider the Lyapunov candidate:

$$
V=\frac{1}{2} s^{T} M(q) s+\frac{1}{2 \eta} \tilde{B}^{T} \tilde{B}
$$

where:

$$
\tilde{B}=\hat{B}-B
$$

Taking the time derivative of (33), we obtain:

$$
\dot{V}=s^{T} M(q) \dot{s}+\frac{1}{2} s^{T} \dot{M}(q) s+\frac{1}{\eta} \tilde{B}^{T} \dot{\hat{B}}
$$

After several mathematical manipulations and following similar steps done in theorem 1 , we can write (35) as:

$$
\begin{aligned}
\dot{V}=s^{T}\left(\tau+f_{\sigma}(q)-C(q, \dot{q}) \dot{q}_{r}-G(q)-\right. & \left.M(q) \ddot{q}_{r}\right) \\
& +\frac{1}{\eta} \tilde{B}^{T} \dot{\hat{B}}
\end{aligned}
$$

Using the control action of (31) and after several simplifications, (36) can be rewritten as:

$$
\begin{gathered}
\dot{V}=s^{T}\left(-\hat{B} \operatorname{sgn}(s)-k_{d} s+f_{\sigma}(q)\right)+\frac{1}{\eta} \tilde{B}^{T} \dot{\hat{B}} \\
\dot{V}=-k_{d} s^{T} s-\left|s^{T}\right| \hat{B}+s^{T} f_{\sigma}(q)+\frac{1}{\eta} \tilde{B}^{T} \dot{\hat{B}}
\end{gathered}
$$

From (14) and (16), we have:

$$
s^{T} f_{\sigma}(q) \leq\left|s^{T}\right| B
$$

Therefore, from (38) and (39), we can have:

$$
\begin{gathered}
\dot{V} \leq-k_{d} s^{T} s-\left|s^{T}\right| \hat{B}+\left|s^{T}\right| B+\frac{1}{\eta} \tilde{B}^{T} \dot{\hat{B}} \\
\dot{V} \leq-k_{d} s^{T} s-\left|s^{T}\right| \tilde{B}+\frac{1}{\eta} \tilde{B}^{T} \dot{\hat{B}} \\
\dot{V} \leq-k_{d} s^{T} s+\tilde{B}^{T}\left(\frac{1}{\eta} \dot{\hat{B}}-|s|\right)
\end{gathered}
$$

Note 2. If the first line of (32) is true, then the term $\tilde{B}^{T}\left(\frac{1}{\eta} \dot{\hat{B}}-\right.$ $|s|)$ of (42) is cancelled out. If the second line of (32) is true, then the term $\tilde{B}^{T}\left(\frac{1}{\eta} \dot{\hat{B}}-|s|\right)$ of (42) would be $\tilde{B}^{T}(-|s|)$ and $\tilde{B}^{T} \geq 0$ since $\hat{B}=M_{B}$ and $M_{B} \geq B$. Therefore, according to (32) we can say that $\tilde{B}^{T}\left(\frac{1}{\eta} \dot{\hat{B}}-|s|\right) \leq 0$, and from (42), we can deduce that:

$$
\dot{V} \leq-k_{d} s^{T} s
$$

Integrating both sides of (43), we get:

$$
\int_{0}^{t} k_{d} s^{T} s \leq V(0)-V(t)<\infty
$$

Likewise to theorem 1 , it can be shown that $s \rightarrow 0$ as $t \rightarrow \infty$. Furthermore, $\hat{B}$ is bounded that leads to deduce that the control action given in (31) is bounded.

Therefore, assumption A2 has been relaxed and there is no need to know the bounds of the functions $f_{\sigma}(q)$. (33) is really a common Lyapunov function for all subsystems of 
the overall switched robotic system. The term $\hat{B} \operatorname{sgn}(s)$ in (31) is compensating for the term $J^{T}(q) D_{\sigma}^{T}(\alpha) \lambda$ caused from the constraints. In spite of the robustness in sliding mode control schemes, it has the drawback of possible chattering that may raise high frequency components and can even cause performance degradation. Therefore, rather than depending on the filtered error $s$, consider the modified filtered error:

$$
s_{\varepsilon}=s-\varepsilon s a t\left(\frac{s}{\varepsilon}\right)
$$

where $\varepsilon$ is a small positive constant and sat(.) is the saturation function. Therefore, for increasing the robustness of the control strategy against possible chattering, let's consider the theorem below.

Theorem 3: For the robotic system described by (7) satisfying assumption A1, the control law:

$\tau=C(q, \dot{q})\left(\varepsilon \operatorname{sat}\left(\frac{s}{\varepsilon}\right)+\dot{q}_{r}\right)+M(q) \ddot{q}_{r}+G(q)-\hat{B} \operatorname{sgn}\left(s_{\varepsilon}\right)-k_{d} s$

and parameters update law:

$\dot{\hat{B}}= \begin{cases}\eta\left|s_{\varepsilon}\right| & \text { if }\left(|\hat{B}|<M_{B}\right) \text { or }\left(\hat{B}=M_{B} \text { and } \eta\left|s_{\varepsilon}\right| \leq 0\right) \\ P\left(\eta\left|s_{\varepsilon}\right|\right) & \text { if }\left(\hat{B}=M_{B} \text { and } \eta\left|s_{\varepsilon}\right|>0\right)\end{cases}$

can guarantee global stable system performance, where $M_{B}$ is a design parameter vector, $\eta, k_{d}>0$ and $P($.$) is the projection$ function, that is:

$$
P\left(\eta\left|s_{\varepsilon}\right|\right)=\eta\left|s_{\varepsilon}\right|-\eta\left|s_{\varepsilon}\right|\left(\frac{\hat{B}^{T} \hat{B}}{|\hat{B}|^{2}}\right)
$$

Proof: Consider the Lyapunov candidate:

$$
V=\frac{1}{2} s_{\varepsilon}^{T} M(q) s_{\varepsilon}+\frac{1}{2 \eta} \tilde{B}^{T} \tilde{B}
$$

From (45), we have $\dot{s}_{\varepsilon}=\dot{s}$. So, taking the time derivative of (48) we obtain:

$$
\dot{V}=s_{\varepsilon}^{T} M(q) \dot{s}+\frac{1}{2} s_{\varepsilon}^{T} \dot{M}(q) s_{\varepsilon}+\frac{1}{\eta} \tilde{B}^{T} \dot{\hat{B}}
$$

Using (7),(11),(13), and after several mathematical manipulations we can have:

$$
\begin{aligned}
\dot{V}= & s_{\varepsilon}^{T}\left(-C(q, \dot{q})\left(s_{\varepsilon}+\varepsilon s a t\left(\frac{s}{\varepsilon}\right)+\dot{q}_{r}\right)-G(q)+\tau\right. \\
& \left.+f_{\sigma}(q)-M(q) \ddot{q}_{r}\right)+\frac{1}{2} s_{\varepsilon}^{T} \dot{M}(q) s_{\varepsilon}+\frac{1}{\eta} \tilde{B}^{T} \dot{\hat{B}}
\end{aligned}
$$

Using the control law of (46), property P2, and after several simplifications, (50) can be written as:

$$
\begin{array}{r}
\dot{V}=s_{\varepsilon}^{T}\left(f_{\sigma}(q)-\hat{B} \operatorname{sgn}\left(s_{\varepsilon}\right)-k_{d} s\right)+\frac{1}{\eta} \tilde{B}^{T} \dot{\hat{B}} \\
\dot{V}=-k_{d} s_{\varepsilon}^{T} s-\hat{B} s_{\varepsilon}^{T} \operatorname{sgn}\left(s_{\varepsilon}\right)+s_{\varepsilon}^{T} f_{\sigma}(q)+\frac{1}{\eta} \tilde{B}^{T} \dot{\hat{B}}
\end{array}
$$

From (14) and (16), we have $s_{\varepsilon}^{T} f_{\sigma}(q) \leq\left|s_{\varepsilon}^{T}\right| B$ and from (45), we have $s=s_{\varepsilon}+\varepsilon \operatorname{sat}\left(\frac{s}{\varepsilon}\right)$. Therefore, we can have:

$$
\begin{aligned}
& \dot{V} \leq-k_{d} s_{\varepsilon}^{T}\left(s_{\varepsilon}+\varepsilon \operatorname{sat}\left(\frac{s}{\varepsilon}\right)\right)-\hat{B}\left|s_{\varepsilon}^{T}\right|+\left|s_{\varepsilon}^{T}\right| B+\frac{1}{\eta} \tilde{B}^{T} \dot{\hat{B}} \\
& \dot{V} \leq-k_{d} s_{\varepsilon}^{T} s_{\varepsilon}-k_{d} s_{\varepsilon}^{T} \varepsilon \operatorname{sat}\left(\frac{s}{\varepsilon}\right)-\hat{B}\left|s_{\varepsilon}^{T}\right|+\left|s_{\varepsilon}^{T}\right| B+\frac{1}{\eta} \tilde{B}^{T} \dot{\hat{B}}
\end{aligned}
$$

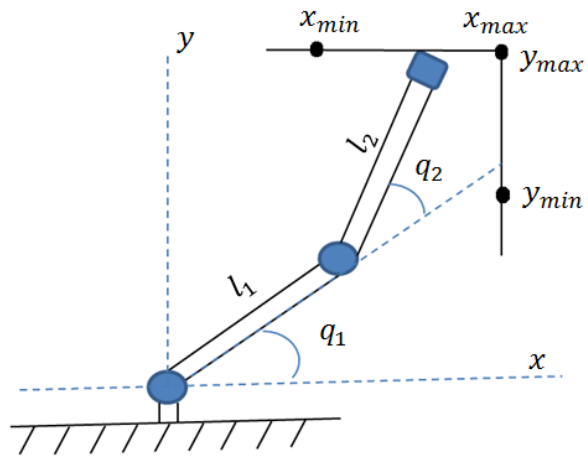

Fig. 1. Two link robotic system interacting with switching constraints.

$$
\begin{gathered}
\dot{V} \leq-k_{d} s_{\varepsilon}^{T} s_{\varepsilon}-k_{d} s_{\varepsilon}^{T} \varepsilon \operatorname{sat}\left(\frac{s}{\varepsilon}\right)-\tilde{B}\left|s_{\varepsilon}^{T}\right|+\frac{1}{\eta} \tilde{B}^{T} \dot{\hat{B}} \\
\dot{V} \leq-k_{d} s_{\varepsilon}^{T} s_{\varepsilon}-k_{d} s_{\varepsilon}^{T} \varepsilon \operatorname{sat}\left(\frac{s}{\varepsilon}\right)+\tilde{B}^{T}\left(\frac{\dot{\hat{B}}}{\eta}-\left|s_{\varepsilon}\right|\right)
\end{gathered}
$$

Similarly to Note 2 mentioned in theorem 2 , we can conclude that $\tilde{B}^{T}\left(\frac{\dot{\hat{B}}}{\eta}-\left|s_{\varepsilon}\right|\right) \leq 0$. Therefore, we can say that:

$$
\begin{gathered}
\dot{V} \leq-k_{d} s_{\varepsilon}^{T} s_{\varepsilon}-k_{d} s_{\varepsilon}^{T} \varepsilon s a t\left(\frac{s}{\varepsilon}\right) \\
\dot{V} \leq-k_{d} s_{\varepsilon}^{T} s_{\varepsilon}
\end{gathered}
$$

Integrating both sides of (58), we obtain:

$$
\int_{0}^{t} k_{d} s_{\varepsilon}^{T} s_{\varepsilon} \leq V(0)-V(t)<\infty
$$

Therefore, we have $s_{\varepsilon} \in \mathcal{L}_{2} \cap \mathcal{L}_{\infty}$ and $\tilde{B} \in \mathcal{L}_{\infty}$. From (45), we can deduce that $s$ is bounded that implies (according to (10)) $\tilde{q}$ is bounded. So, from (9) and assumption A1, we can say that $q$ is also bounded. According to (11), we have $s$ is bounded. Furthermore, from (7), (13), and assumption A1 we can conclude that $\dot{s}$ is bounded, that is $\dot{s} \in \mathcal{L}_{\infty}$ which implies that $\dot{s}_{\varepsilon} \in \mathcal{L}_{\infty}$. Therefore, according to Barbalats lemma, we can have $s_{\varepsilon} \rightarrow 0$ as $t \rightarrow \infty$ that makes $\tilde{q} \rightarrow \Omega_{\varepsilon}$ as $t \rightarrow \infty$. The region of attraction $\Omega_{\varepsilon}$ can be made small by properly choosing the values of $\varepsilon$ and $\gamma$.

\section{Simulation Results}

We will consider the case of a two link robotic system interacting with an environment composed of two orthogonal lines, the first one is parallel to the $x$-axis and the other one is parallel to the $y$-axis. Fig. 1 shows the configuration of the robotic system considered throughout this simulation. The end effector is required to move along the constraints with bounds of $x_{\min }$ and $x_{\max }$ along the constraint parallel to the $x$-axis and $y_{\min }$ and $y_{\max }$ along the constraint parallel to the $y$-axis. The end effector velocity along the $x$ and $y$ axes is assumed to be constant. The dynamic equations of motion of the robotic system given in Fig. 1 can be described by (6) with [10]:

$$
M(q)=\left(\begin{array}{cc}
\left(m_{1}+m_{2}\right) \ell_{1}^{2} & m_{2} \ell_{1} \ell_{2}\left(s_{1} s_{2}+c_{1} c_{2}\right) \\
m_{2} \ell_{1} \ell_{2}\left(s_{1} s_{2}+c_{1} c_{2}\right) & m_{2} \ell_{2}^{2}
\end{array}\right)
$$




$$
\begin{gathered}
C(q, \dot{q})=m_{2} \ell_{1} \ell_{2}\left(c_{1} s_{2}-s_{1} c_{2}\right)\left(\begin{array}{cc}
0 & -\dot{q}_{2} \\
-\dot{q}_{1} & 0
\end{array}\right) \\
G(q)=\left(\begin{array}{c}
-\left(m_{1}+m_{2}\right) \ell_{1} g s_{1} \\
-m_{2} \ell_{2} g s_{2}
\end{array}\right)
\end{gathered}
$$

The masses of the links $m_{1}$ and $m_{2}$ are assumed to be of $1(\mathrm{~kg})$. The links length $\ell_{1}$ and $\ell_{2}$ will be considered to be of $1(\mathrm{~m})$. The acceleration due to gravity $g=9.8\left(\mathrm{~m} / \mathrm{s}^{2}\right)$. According to theorem 3 , the whole term $J^{T}(q) D_{\sigma}^{T}() \lambda$ is not required to be known. We will consider the velocity along the first (that is parallel to the $x$-axis) and second (that is parallel to the $y$-axis) constraints to be $0.24(\mathrm{~m} / \mathrm{s})$. The motion of the end effector will start from the point $x_{\min }$ on the first constraint and move along the constraint towards the point $x_{\max }$ and as soon as the effector reaches to $x_{\max }$ it switches to move along the second constraint heading towards $y_{\min }$. When the end effector reaches to $y_{\min }$, it reverses its direction of movement and moves towards $y_{\max }$ along the second constraint. As soon as the end effector reaches to $y_{\max }$, it switches to move along the first constraint towards $x_{\min }$ and when it reaches $x_{\min }$ the whole cycle of movement is repeated. In our simulation we took the values of $x_{\min }$ and $x_{\max }$ to be $0.3(\mathrm{~m})$ and $0.5(\mathrm{~m})$ respectively. While $y_{\min }$ and $y_{\max }$ were considered to be $1.5(\mathrm{~m})$ and $1.2(\mathrm{~m})$ respectively. The control algorithm given in theorem 3 has been used with $M_{B}=\left[\begin{array}{ll}0.2 & 0.2\end{array}\right]^{T}, k_{d}=30$ and $\eta=1$. For the values of $\gamma$ and $\varepsilon$ and after several trials we came up with values of $\gamma=10$, and $\varepsilon=0.01$. The desired $x$ and $y$ positions were successfully tracked by the end effector with the constraints been continuously switched between the first and the second constraints. Fig. 2 (a) show the desired and actual position of link1 and Fig. 3 (a) show the corresponding signals for link 2 . The desired and actual velocity signals of link 1 are given in Fig. 2 (b) and that of link2 are shown in Fig. 3 (b). Fig. 4 (a) and (b) show the angular position error signals for link1 and link2 respectively. The error signals of the $x$ and $y$ coordinates of the end effector are given in Fig. 5 (a) and (b) respectively. Fig. 6 (a) and (b) illustrate the modified filtered error of link1 and link2 respectively. The upper bounds estimations for both links are given in Fig. 7 and finally, we have the torque signals on each link is given in Fig. 8. It is obvious from the figures above that an excellent tracking performance was obtained despite the switching in the constraints. If we decrease $\varepsilon$ and/or increase $\gamma$, then the region of attraction $\Omega_{\varepsilon}$ can be reduced which improves the tracking error. However, reducing the region of attraction would increase the chattering [20,21], therefore the choice of $\varepsilon$ and $\gamma$ should be done such that a compromise solution is obtained. Even though the signals $q_{d}$ and $\dot{q}_{d}$ are piecewise continuous, they have several hard edges at certain instants that causes the filtered and consequently the torque signals to be suffering from spikes at those instants. However, such spikes are not affecting the system performance as can be seen from the error signals shown in Fig. 4 and 5.

\section{CONClusion}

In this paper, a robust control strategy is presented to robotic systems with switched constraints. The derivation is started with suggesting a sliding mode control strategy that can guarantee global stable performance of the given system. However, the bounds of the functions caused from the constraints are assumed to be known. The assumption of knowing bounds
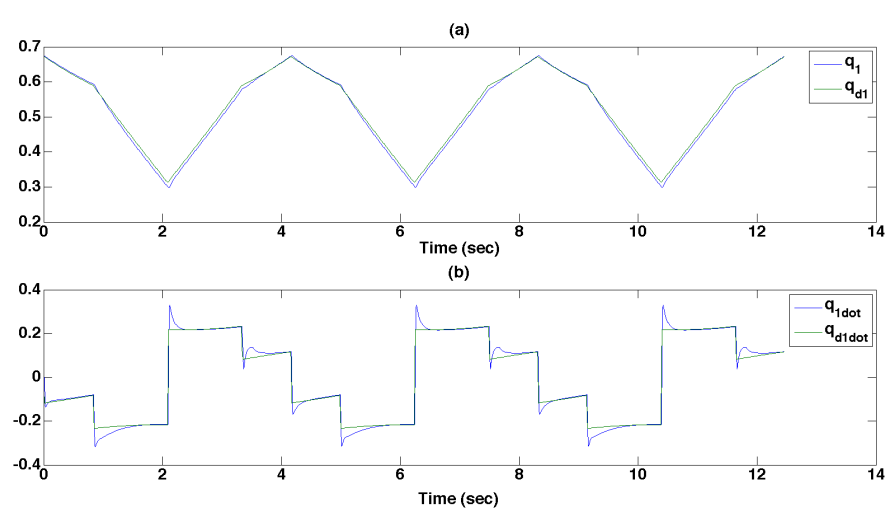

Fig. 2. (a) Link1 desired and actual angular positions in rad. (b) Link1 desired and actual angular velocities in $\mathrm{rad} / \mathrm{sec}$.
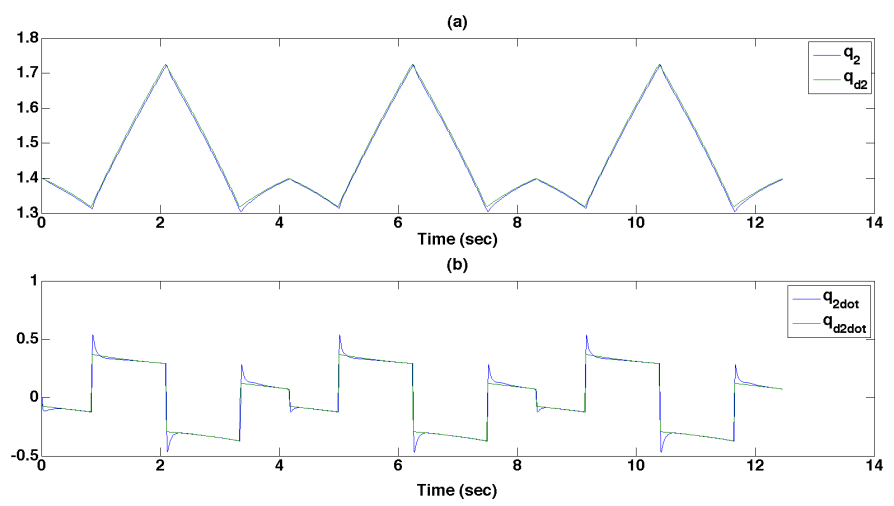

Fig. 3. (a) Link2 desired and actual angular positions in rad. (b) Link2 desired and actual angular velocities in $\mathrm{rad} / \mathrm{sec}$.
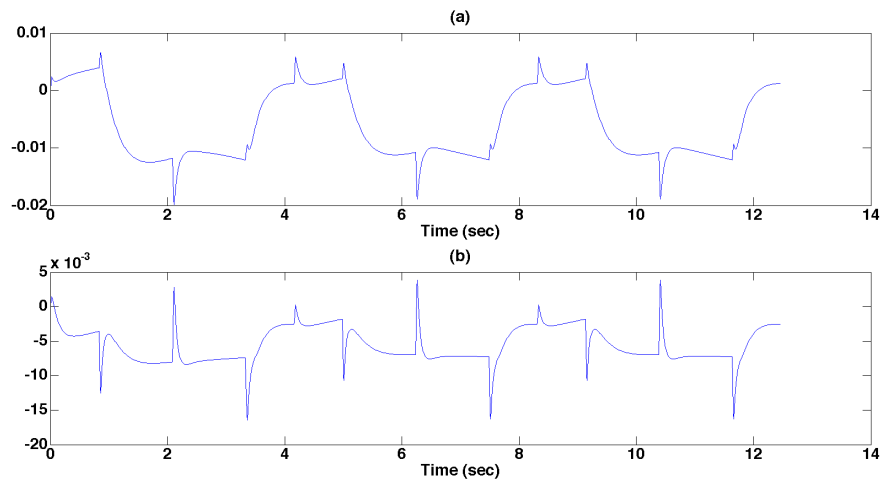

Fig. 4. (a) Link1 angular position error signal(b) Link2 angular position error signal.
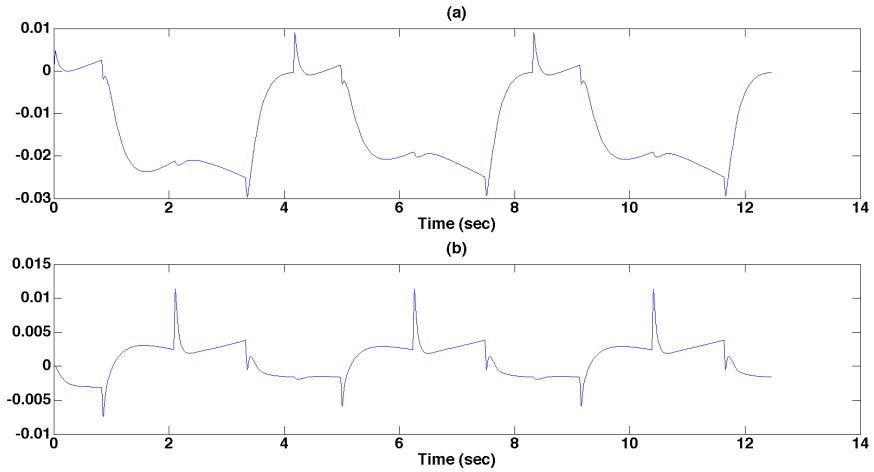

Fig. 5. (a) End effector $x$-coordinate error signal.(b) End effector $y$-coordinate error signal. 
(a)

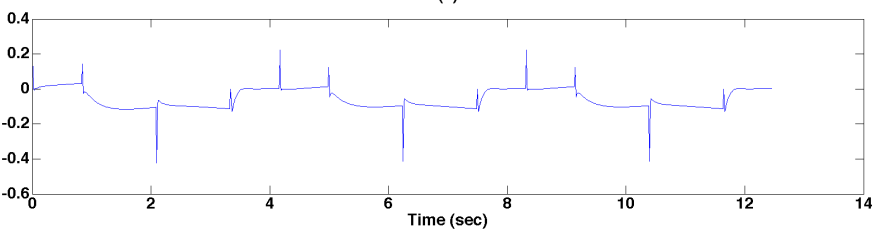

(b)

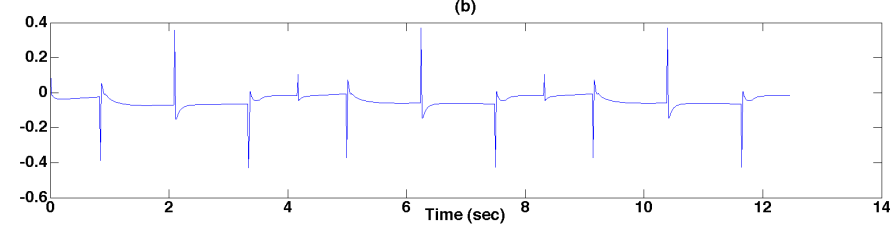

Fig. 6. (a) Link1 modified filtered error signal.(b) Link2 modified filtered error signal.
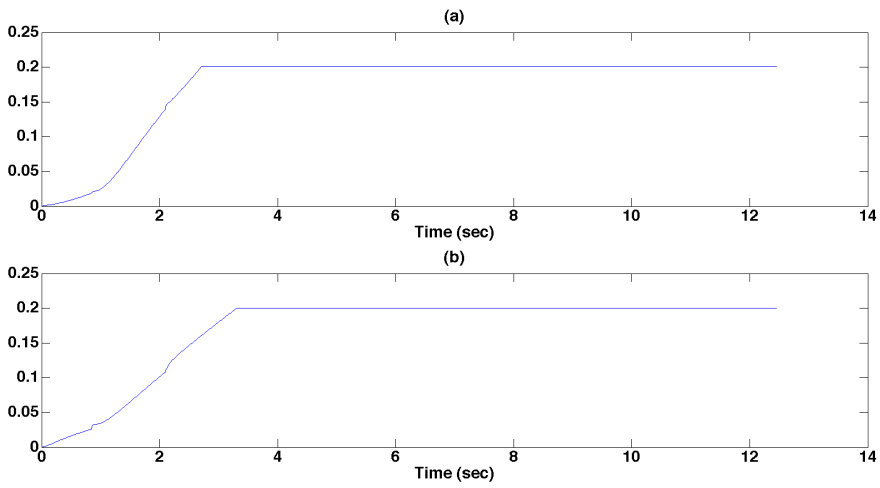

Fig. 7. (a) $\hat{B}_{1}$ :Constraint upper bound estimation on link1.(b) $\hat{B}_{2}$ :Constraint upper bound estimation on link2.

of the constraints function is then relaxed through suggesting an adaptive sliding mode control strategy that is proved to guarantee global stability for the switched constrained robotic system. Possible chattering is then reduced through improving the suggested adaptive sliding mode control strategy with presenting a modified filtered error and deriving the control and parameter update laws with respect to this new filtered error. Simulation was performed for a two link robotic system interacting with two switched constraints. From the simulation excellent tracking performance can be noticed. Despite the excellent tracking performance of the suggested approach, the parameters of the robot dynamics are required to be precisely known and future works should focus on relaxing the need for knowing those parameters for the switched constrained robots. Possible use of the fuzzy control strategy can achieve such a purpose; however this should be left to the emphasis of future works.
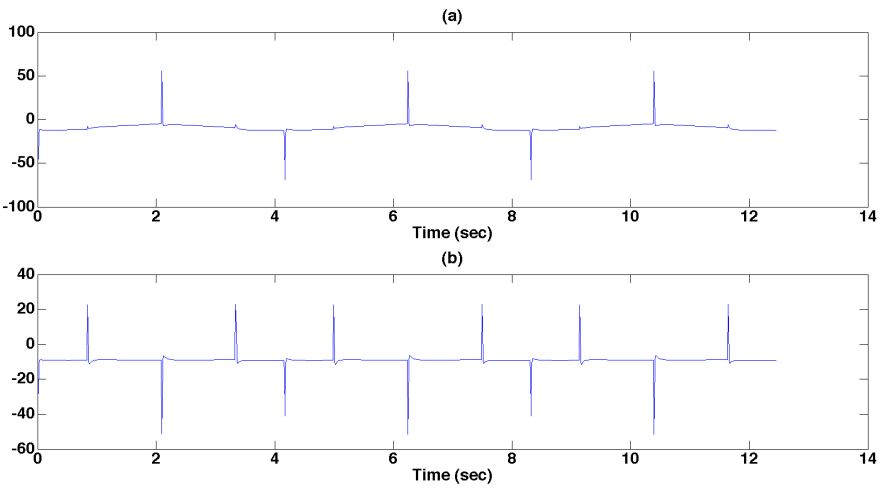

Fig. 8. (a) Link1 control action $\tau_{1}$ in N.m.(b) Link2 control action $\tau_{2}$ in N.m.

\section{ACKNOWLEDGMENT}

This work is partially supported by the Fonds Nationale de la Recherche (FNR) under grant no. AFR 2955286.

\section{REFERENCES}

[1] M. Takegaki, and S. Arimoto, "A new feedback method for dynamic control of manipulators," Trans. of ASME, J. of Dyn. Syst., Meas. and Contr., vol. 103, no. 2, 1981, pp. 119- 125.

[2] P. Tomei, “ Adaptive PD controller for manipulators," IEEE Trans. Robot. and Autom., vol. 7, no. 4, 1991, pp. 233- 243.

[3] J. J. Slotine, and W. Li, " On adaptive control of robot manipulators," Int. J. Robot. Res., vol. 6, 1987, pp. 565- 570.

[4] K.W. Lee and H.K. Khalil, "Adaptive output feedback control of robot manipulators using high-gain observers," Int. J. Control, vol. 67, 869886, 1997.

[5] M. H. Raibert, and J. J. Craig, "Hybrid position/force control of manipulators," Trans. of ASME, J. of Dyn. Syst., Meas. and Contr., vol. 102, 1981, pp. 126- 133.

[6] D. Wang, and N. H. McClamroch, "Position and force control for constrained manipulator motion: Lyapunovs direct method," IEEE Trans. Robot. and Autom., vol. 9, no. 3, 1993, pp. 308- 313.

[7] G. Song, L. Cai, " Robust position/force control of robot manipulators during constrained tasks," IEE Proc. Contr. Theor. Appl., vol. 145, no. 4, 1998, pp. 427- 433.

[8] H. Krishnan, and N. H. McClamroch, "Tracking in nonlinear differentialalgebraic control systems with applications to constrained robot systems," Automatica, vol. 30, no. 12, 1994, pp. 1885- 1897.

[9] Y. C. Chang, B. S. Chen, "Robust tracking designs for both holonomic and nonholonomic constrained mechanical systems: adaptive fuzzy approach," IEEE Trans. Fuzz. Syst., vol. 8, no. 1, 2000, pp. 46- 66.

[10] M. W. Spong,S.Hutchinson, and M. Vidyasagar, Robot Modeling and Control, John Wiley and Sons,Inc.,2006.

[11] A. Albu-Schäffer, C. Ott, and G. Hirzinger, "A unified passivity- based control framework for position, torque and impedance control of flexible joint robots," Int. J. on Robot. Res., vol. 26, no. 1, 2007, pp. 23- 39.

[12] P. R. Quyang, W. J. Zhang, and M. M. Gupta, "An adaptive switching learning control method for trajectory tracking of robot manipulators," Mechatronics, vol. 16, 2006, pp. 51- 61.

[13] P. R. Pagilla, and Y. Zhu, "Adaptive control of mechanical systems with time- varying parameters and disturbances," Trans. of ASME, J. of Dyn. Syst., Meas. and Contr., vol. 26, 2004, pp. 520- 530.

[14] I. F. Jasim, and N. F. Jasim, "Adaptive sliding mode control design for a class of nonlinear systems with unknown deadzone of unknown bounds," Proc. 2010 1st Int. Conf. on Energy, Power, and Control, Basrah- Iraq, 30 Nov- 2 Dec, 2010, pp. 7- 11.

[15] I. F. Jasim, "Robust adaptive controller and observer design for a class of nonlinear systems with unknown backlash- like hysteresis," IEEE Int. Conf. on Contr. Syst., Comput. and Eng., Penang- Malaysia, 25- 27 Nov., 2011, pp. 187- 192.

[16] I. F. Jasim, "Improved observer- based robust adaptive control for a class of nonlinear systems with unknown deadzone," Proc. IMechE Part I: J. of Syst. and Contr. Eng., vol. 227, no. 2, 2013, pp. 184- 197.

[17] D. Liberzon, Switching in Systems and Control, Birkuser, 2003.

[18] J. K. Mills, "Constrained manipulator dynamic models and hybrid control," Proc. 1988 IEEE Int. Symp. on Intell. Contr., Arlington- VA, 24- 26 August, 1988, pp. 424429.

[19] N. H. McClamroch, "Singular systems of differential equations as dynamic models for constrained robot systems," Proc. 1986 IEEE Int. Conf. Robot. and Autom., San Francisco- CA, 8- 10 April, 1986, pp.2128.

[20] J.- J. E. Slotine, "Sliding controller design for nonlinear systems," Int. J. Control, vol. 40, 1984, pp. pp. 435-448.

[21] J.- J. E. Slotine, and J. A. Coestee, "Adaptive sliding control synthesis for nonlinear systems," Int. J. Control, vol. 43, 1986, pp. 1631- 1651. 